\title{
Neurological complications of human immunodeficiency virus infection
}

\author{
Peter G.E. Kennedy \\ Glasgow University Department of Neurology, Institute of Neurological Sciences, Southern General Hospital, \\ Glasgow G51 4TF, UK.
}

\begin{abstract}
Summary: The protean neurological manifestations of human immunodeficiency virus (HIV) infection are reviewed. Both the central nervous system and peripheral nervous system may be affected and many of the complications may occur in individuals with acquired immunodeficiency syndrome (AIDS)-related complex, or who are seropositive for HIV alone as well as those with the established AIDS syndrome. Specific therapy is available for certain of these neurological conditions, but the clinical course in others is untreatable and progressive. Although it seems likely that the pathogenesis of some of these syndromes such as the AIDS-dementia complex are due to the direct effect of HIV on the nervous system, in others the neurological injury probably occurs as a consequence of the immunosuppression which HIV induces, or immune-mediated mechanisms.
\end{abstract}

\section{Introduction}

Human immunodeficiency virus (HIV) is the causative retroviral agent of the acquired immunodeficiency syndrome (AIDS). Previously known as lymphadenopathy-associated virus $(\mathrm{LAV})^{1}$ or human T-lymphotropic virus III (HTLV-III) ${ }^{2}$, the term HIV has now gained widespread acceptance. Neurological involvement in AIDS is common. In early studies from New York and San Francisco it appeared that $30-40 \%$ of patients with AIDS became neurologically symptomatic during their illness, ${ }^{3,4}$ and approximately $10 \%$ of patients presented with neurological symptoms. ${ }^{4}$ However, neuropathological evidence of involvement of the central nervous system (CNS) has indicated that $55-80 \%$ of patients dying with AIDS have pathological abnormalities in their CNS.4, 5,6 More recent clinical studies have indicated that neurological involvement in AIDS is indeed more common than previously thought with two-thirds of patients having some kind of neurological abnormality, in particular evidence of the AIDSdementia complex. ${ }^{7}$ The estimated percentage of patients with CNS involvement depends also on the severity of the symptoms. For example, as many as $90 \%$ of individuals probably have evidence of the

Correspondence: Professor P.G.E. Kennedy, M.D., Ph.D., M.R.C.P.

Accepted: 15 October 1987
AIDS-dementia complex if only mildly affected patients are included. ${ }^{7}$ Our own experience in Glasgow (Kennedy et al., in preparation) is in agreement with a high incidence of neurological complications, and the picture will become clearer with time and when more autopsy examinations are carried out on patients dying with AIDS.

The spectrum of neurological disease occurring in AIDS patients is very wide. The CNS is most frequently affected but a number of peripheral nervous system (PNS) syndromes have also been recognized. There appear to be at least two general pathogenetic mechanisms of infectious disease of the CNS in AIDS, namely, direct involvement of HIV in some conditions, and in others opportunistic infections with organisms that do not usually cause disease in normal immunocompetent individuals occur. These organisms appear to become pathogenic in AIDS patients as a result of the immunosuppressive state which the virus induces. HIV causes a severe defect in cell-mediated immune mechanisms through its selective infection of the $T$ helper lymphocyte population ${ }^{8}$ which plays a central role in the immune response. These two mechanisms of neurological damage are not mutually exclusive and in some cases both HIV and opportunistic organisms such as cytomegalovirus (CMV) may be pathogenic. In other cases more than one opportunistic infection, e.g., CMV and

(C) The Fellowship of Postgraduate Medicine, 1988 
herpes simplex virus (HSV) may operate. ${ }^{9}$ An additional factor which has emerged over the last few years is that CNS and PNS complications may occur not only in patients with full-blown AIDS but also in those with the AIDS-related complex (ARC), or even asymptomatic individuals who are only seropositive for HIV.10,11 The long-term prognosis of such patients remains to be seen but both host and viral factors must almost certainly interact to produce these heterogeneous patterns of disease.

The following account will provide an overview of the neurological complications seen in AIDS patients and will not be limited to infections, although infectious agents including HIV acting directly by itself may well prove eventually to be implicated in virtually all of these nervous system manifestations. Emphasis will be placed on the more common conditions such as the AIDSdementia complex. The complications will be considered under four broad groups: (a) infections of the CNS, (b) neoplasms, (c) vascular disturbances and (d) PNS complications.

\section{Infections of the CNS (Table I) \\ AIDS-dementia complex}

Since the early AIDS cases were first encountered it had been recognized that affected patients were frequently slow mentally and had difficulty concentrating. These symptoms usually heralded a progressive dementia which was initially called 'subacute encephalitis'4 but has recently been

Table I Neurological complications of HIV infectionCNS complications

(a) Infections

AIDS-dementia complex

CMV encephalitis

HSV and VZV encephalitis

Cerebral toxoplasmosis

Progressive multifocal leukoencephalopathy

Vacuolar myelopathy and myelitis

Aseptic meningitis

Fungal infections

Mycobacterial infections

Treponema infection

CMV retinitis

(b) Neoplasms

Primary CNS lymphoma

Systemic CNS lymphoma

Kaposi's sarcoma

(c) Vascular

Cerebral haemorrhage

Cerebral infarction termed the 'AIDS-dementia complex'. ${ }^{10}$ This is now recognized as being the commonest neurological manifestation of AIDS occurring in at least two thirds of affected patients, ${ }^{7}$ and, as has been indicated above, the true incidence may be very much greater if mild cases are included. Moreover, nearly $25 \%$ of cases with this condition occur in patients with seropositivity for HIV alone or ARC before the full-blown AIDS syndrome develops. ${ }^{10}$

The syndrome is usually progressive over a period of months and the initial complaints are of lethargy, difficulty with concentration and memory disturbance. A confusional state including organic psychosis may develop and personality change with general apathy may also be evident. Motor features may develop early or later in the illness. The latter include weakness, ataxia, tremor, impaired ocular motility and myoclonus; ${ }^{7}$ seizures may also occur. A variety of signs may be detected, slurring of motor and verbal responses, hyper-reflexia, frontal lobe release signs and the neuropsychological features of a subcortical dementia being the typical findings. ${ }^{7}$ The clinical course is usually relentless, resulting in increasing dementia until the patient is bed-bound and incontinent. Some of the earlier signs, e.g., frontal lobe abnormalities, may be subtle and only detected if specifically looked for (Kennedy et al., in preparation ${ }^{7}$ ). In children with AIDS the progressive encephalopathy may be characterized by loss of motor milestones or intellectual abilities, secondary microcephaly, seizures and myoclonus. ${ }^{12,13}$

Neurological investigations may reveal a variety of abnormalities. The cerebrospinal fluid (CSF) usually shows non-specific features such as a mild pleocytosis and raised protein. HIV has been isolated from the CSF of both patients with the AIDS-dementia complex and those with other CNS syndromes, ${ }^{14}$ and locally synthesized HIV-specific IgG has been detected in the CSF in such patients. ${ }^{15}$ Computed tomographic (CT) scanning in the AIDS-dementia complex usually shows cortical atrophy with consequent enlargement of the ventricles, ${ }^{10}$ sometimes associated with basal ganglion calcification in children. ${ }^{12}$ In our own experience (Kennedy et al. in preparation) and that of others ${ }^{7}$ the atrophic changes seen on CT scanning may occur at an early stage of the dementia. Magnetic resonance imaging (MRI) abnormalities consisting of white matter attenuation have also been demonstrated in these patients. ${ }^{7}$

Characteristic pathological findings in patients who have died with the AIDS-dementia complex include diffuse small areas of focal perivascular demyelination in white matter, ${ }^{3}$ diffuse microglial nodules in grey and white matter, ${ }^{4,16}$ reactive 
astrocytosis, ${ }^{4}$ focal or diffuse white matter vacuolation $^{7}$ and accumulation of perivascular macrophages. ${ }^{4,7}$

Earlier studies suggested that CMV may be the causative agent of the syndrome in view of the characteristic histopathology, ${ }^{4,16}$ the isolation of CMV from some of the patients' brains ${ }^{4}$ and the demonstration of CMV antigens in brain tissue. ${ }^{13}$ Although it is possible that a true CMV encephalitis may indeed occur in AIDS, it now seems likely that the AIDS-dementia complex results from the direct effect of HIV in the brain. The initial convincing evidence for this view was provided by a study which used Southern blotting analysis and in situ molecular hybridization to demonstrate the presence of HIV DNA in the brains of five patients; viral-specific RNA was also detected in four of these individuals. ${ }^{17}$ These findings have now been corroborated by several other studies. For example, HIV has been isolated from the CSF and a variety of neural tissues such as brain from AIDS patients with neurological involvement including those with dementia. ${ }^{14,18}$ In addition, the presence of HIV nucleic acid sequences and proteins has been confirmed in the brains of these patients using in situ hybridization and immunocytochemistry. ${ }^{19}$ The viral genome was localized mainly in capillary endothelial cells, mononuclear inflammatory cells and giant cells. Very recently, the presence of HIV proteins in these brains has been confirmed using immunocytochemistry. ${ }^{20}$ Although there is some evidence that HIV may be present in some glial cells and neurones, ${ }^{19,20}$ the evidence for this is not unequivocal. Although the virus is clearly present in macrophages, in the author's opinion the precise glial cell localization of HIV has yet to be determined convincingly. It should also be borne in mind that the dementia may be a consequence of an infection with more than one virus and both HIV and CMV (or another virus) may be acting synergistically to produce the neurological disease. By contrast, a condition in AIDS which has been shown unequivocally to be due to CMV is CMV chorioretinitis leading to blindness in many cases. ${ }^{21}$ A recent study has suggested a further possible mechanism of HIV-mediated neurological disease in that non-cytocidal natural variants of HIV have been isolated from four patients with AIDS and CNS manifestations. ${ }^{22}$ Most of the HIV isolates from end-stage AIDS, however, have been cytocidal to T4 cells. The authors have therefore suggested that the neurological disorders may result from the non-cytocidal HIV variants causing metabolic abnormalities of neural cells rather than direct neural cell killing. There is no specific treatment for the AIDS-dementia complex although it is possible that it may respond to drugs currently being evaluated in AIDS patients such as azidothymidine. ${ }^{23}$

\section{Encephalitis due to herpesviruses}

Encephalitis due to both HSV and varicella-zoster virus (VZV) have been described in patients with AIDS. VZV infections are well-known to occur with increased frequency in immunocompromised individuals, ${ }^{24}$ and encephalitis and arteritis due to VZV may rarely occur in AIDS patients. ${ }^{3,4,7}$ Although HSV encephalitis has been described in these patients ${ }^{4,25}$ this is a rare occurrence. Most cases have been due to HSV-1 encephalitis as would be expected since HSV-2 usually causes encephalitis only in neonates. However, brain biopsy-proven encephalitis due to HSV-2 in two homosexual men with persistent lymphadenopathy has been described. ${ }^{26}$ This is perhaps not surprising in view of the increased incidence of HSV-2 encephalitis in immunosuppressed patients. The clinical picture of HSV encephalitis in AIDS is similar to that occurring in immunocompetent individuals and specific treatment should be given with intravenous acyclovir.

\section{Spinal cord and meningeal conditions}

A vacuolar myelopathy occurring in 20 out of 89 consecutive patients with AIDS has been described, ${ }^{27}$ and it is now clear that about one third of all adult individuals with AIDS have this complication. ${ }^{28}$ The clinical presentation is characterized by spastic paraparesis, ataxia and sphincter disturbance, and dementia is present in many cases. Indeed this myelopathy may be part of the AIDS-dementia complex although its precise relation to the latter entity is not certain. ${ }^{7}$ There does not seem to be a correlation between the incidence and severity of the dementia and the myelopathy. ${ }^{27}$ There is a very typical pathological picture consisting of symmetrical vacuolation in the posterior and lateral columns of the spinal cord, mainly in the thoracic segments. Some patients also have similar vacuolation within the brain. Electron microscopic analysis has demonstrated swelling within myelin sheaths which appeared to lead to the vacuolar changes. Some of the vacuoles contained lipid-laden macrophages. The aetiology of this condition is not known. The pathological changes are similar in many respects to those seen in subacute combined degeneration of the cord due to $B_{12}$ and folate deficiency. However, there are clinical differences between the two groups of 
patients and the serum $B_{12}$ levels are normal in the AIDS patients with the syndrome. ${ }^{27}$ Whether HIV is directly involved in the pathogenesis of the myelopathy is unknown.

Spinal cord degeneration in an individual with AIDS was also described by other authors ${ }^{29}$ and this patient may have had the same condition. Symmetrical degenerative spongy changes and demyelination were seen in the lateral and anterior pyramidal tracts and the posterior columns, and scattered microglial nodules were seen in the spinal cord and brain. The aetiology of this condition was, again, unclear.

Inflammatory conditions of the spinal cord and meninges are also well-described in AIDS. For example, aseptic meningitis is a recognized feature which may occur at the time of HIV seroconversion. The aseptic meningitis in AIDS presents in a different clinical way from the AIDS-dementia complex. ${ }^{4}$ Fever, headache and meningism are typical and the attacks tend to be self-limiting or recurrent; CMV has been isolated from the CSF of at least one case with this condition. ${ }^{4}$

Ascending myelitis has also been described in AIDS patients. For example, a case of AIDS with Kaposi's sarcoma and disseminated CMV infection who also developed a progressive necrotizing ascending myelitis has been reported. ${ }^{10}$ Both CMV and HSV-2 were isolated from multiple sites throughout the nervous system and CMV was also cultured from the CSF. The myelitis was presumably due to one or both of these viruses. At least two other cases appear to have had myelitis, ${ }^{4}$ and VZV is probably also capable of causing a viral myelitic syndrome. ${ }^{7}$

\section{Progressive multifocal leukoencephalopathy (PML)}

$\mathrm{PML}^{30}$ is a rare but well-recognized opportunistic CNS infection caused by a papovavirus, usually JC virus $^{31}$ but in two cases the SV40 virus ${ }^{32}$ has been implicated. PML occurs in immunosuppressed individuals with defective cell-mediated immunity such as those with Hodgkin's disease and/or who are receiving immunosuppressive drugs. PML is now well-established as an unusual complication of AIDS, $2 \%$ of such patients showing the neuropathological changes of PML at post-mortem. ${ }^{5,7}$ Several cases with the typical clinical features of this condition have now been described. The clinical presentation of PML is characteristic and consists of a steadily progressive illness with mental deterioration and a wide variety of focal disturbances such as blindness, aphasia, hemiparesis, sensory impairment and ataxia. ${ }^{4,7}$ Most patients die within months of the onset.
Investigations in PML are also characteristic, the CT scan usually showing single or, more commonly, multiple low-density lesions in white matter. As might be expected from its greater sensitivity MRI scanning is particularly useful in detecting such lesions at an early stage of the illness. ${ }^{7}$ The CSF is generally normal but the EEG is usually abnormal. The typical pathological findings consist of focal areas of demyelination, absence of inflammatory reaction, enlarged bizarre shaped astrocytes and papovavirus particles within enlarged oligodendrocytes demonstrable by electron microscopy. ${ }^{33}$ By contrast, neurones appear to be unaffected by the disease process on the basis of morphology. There is no specific treatment for PML although both adenine arabinoside ${ }^{34}$ and cytosine arabinoside ${ }^{3}$ have been given to some patients without marked effect.

\section{Cerebral toxoplasmosis}

Toxoplasmosis results from infection with the protozoan parasite Toxoplasma gondii and is one of the commonest opportunistic infections in AIDS patients, being the most frequent cause of focal neurological disease. Indeed, toxoplasmosis is the most frequent cause of CNS disease in Haitian AIDS patients ${ }^{35}$ and accounts for $10-15 \%$ of neurological complications in AIDS overall. ${ }^{28}$ However, this high incidence has not been found in all series, one recording, for example, only one case out of 29 studied at post-mortem. ${ }^{6}$ The reasons for such marked discrepancies are not clear but must relate at least in part to geographical differences.

The clinical picture may be variable in terms of onset, severity and symptomatology. The typical presentation is with focal deficits and altered mentation and consciousness. Seizures are common and may be a presenting feature in about $15 \%$ of cases. ${ }^{4}$ Both fever and headache are common and rarely cerebellar and brain stem involvement may be evident. ${ }^{7}$

CSF analysis in these patients may show nonspecific pleocytosis and elevation of protein levels. Serological tests for toxoplasma IgG are generally unreliable and are not really helpful in diagnosis. False-negative results are well-recognized and IgM toxoplasma titres may be negative in patients with cerebral toxoplasmosis complicating AIDS.,35 Both CT scanning and MRI are extremely useful in diagnosis and typically reveal single or multiple contrast-enhancing ring lesions. The lesions have a special tendency to occur in deep grey matter structures. $^{3}$ Less frequently non-enhancing or homogeneously-enhancing lesions may be seen or the CT scan may be normal..$^{3,4,7,36}$ In all these 
cases differentiation from other causes of focal brain disease such as cerebral lymphoma must be made. MRI appears to be more sensitive than CT in detecting the presence and nature of toxoplasmosis lesions. ${ }^{4,7}$

Treatment of cerebral toxoplasmosis is with pyrimethamine and sulphadiazine which is highly effective in many cases. It is important to start treatment as soon as possible after the clinical diagnosis has been made. Marked clinical improvement may occur many days prior to resolution of the CT appearances ${ }^{7}$ but the relapse rate is still high. There is no general consensus on the exact role of brain biopsy in toxoplasmosis. The author shares the view of some authors ${ }^{7,37}$ which favours a therapeutic trial of pyrimethamine and sulphadiazine in these patients while recommending biopsy for patients who fail to respond to medical therapy or in whom there exists diagnostic doubt. However, other workers have recommended brain biopsy of all AIDS patients who have such mass lesions in view of the unhelpful serology and the fact that more than one infectious intracranial pathology may exist in these patients. ${ }^{4}$

\section{Fungal infections}

CNS fungal infections with Cryptococcus neoformans, Candida albicans, Aspergillus fumigatus, and Coccidiomycosis have all been described in patients with AIDS. The most important of these organisms is Cryptococcus neoformans which is a frequent fungal infection of the CNS in immunocompromised individuals. $^{4,38}$ The primary site of infection is usually the lung with subsequent spread via the bloodstream to the CNS. ${ }^{6}$ Cryptococcal meningitis has been frequently described in patients with AIDS. The presentation is variable and often subacute. Typically, there is the development of meningitic symptoms and signs or the development of raised intracranial pressure. Headache may precede the development of clinical signs by weeks. ${ }^{38}$ Features such as altered consciousness and personality, visual loss, seizures, cranial nerve palsies and the development of long tract signs and hydrocephalus may also be apparent. ${ }^{38}$ Intracranial cryptococcoma has also rarely been described in AIDS patients. ${ }^{4,35}$

Diagnostic studies in suspected cryptococcal meningitis should always include a chest X-ray but this is frequently normal. The CT scan may show cerebral atrophy, white matter changes, hydrocephalus, an intracranial mass, or it may be normal. ${ }^{4.38}$ The CSF is often normal but it may show non-specific changes such as mononuclear pleocytosis, mild protein elevation and depressed glucose levels. The diagnosis on the CSF may be made by visualization of the organisms by Indian ink staining, by means of culture of the organism, or by demonstration of cryptococcal antigen in the CSF. The latter test utilizes a latex agglutination procedure and is of considerable diagnostic value in cryptococcal meningitis. ${ }^{39}$

The treatment of cryptococcal meningitis has usually been with amphotericin $B$ or 5fluorocytosine and the importance of early treatment before the development of major CNS complications has been emphasized. ${ }^{38,40}$ In some cases the use of an Ommaya reservoir for direct intraventricular administration of drugs has been advocated $^{41}$ but this is not without complications. Hydrocephalus should also be treated if present. The prognosis of cryptococcal meningitis is poor even after appropriate treatment with reported mortality rates in the region of $40 \% .^{4}$

Rare cases of Candida albicans infection in AIDS patients have been described, and both meningitis and brain abscesses may occur. ${ }^{\mathbf{3} 42}$ Cerebral abscess due to aspergillosis infection has also been reported. ${ }^{43}$

\section{Other infections}

Syphilitic infections due to Treponema pallidum have been described in AIDS patients and the presentation may be with meningovascular syphilis. ${ }^{43}$ However, it is important to establish that the infection is an active one since many AIDS patients are likely to have had an increased risk of contracting a previous and currently inactive infection. Patients with AIDS also have an increased incidence of CNS Mycobacterium tuberculosis infections, in particular with the atypical Mycobacterium avium intracellulare. The presentation may be with tuberculous meningitis or, more rarely, intracerebral tuberculoma. ${ }^{35}$

\section{Neoplasms (Table I)}

The incidence of malignant neoplasms is increased in patients with AIDS presumably as a result of the immunocompromised state of these patients. CNS lymphoma is the most frequently described malignancy, and a number of cases of primary CNS lymphoma and systemic lymphoma with CNS involvement have been described., ${ }^{3,43}$ Primary lymphoma may be discovered at autopsy without a history of clinical symptomatology. In symptomatic cases the presentation is variable, although there is a marked tendency for the primary lymphoma to involve the brain parenchyma. ${ }^{4}$ The clinical features 
include encephalopathy with alteration of mental status, headaches, seizures, aphasia and focal limb signs such as hemiparesis. In addition, single or multiple cranial neuropathies and intrinsic brain stem syndromes may occur. ${ }^{43}$ The CT scan is often helpful in diagnosis and may show single or multiple lesions, but in some cases it may be normal or show only cerebral atrophy. Pathologically these lymphomas are of the B cell type. ${ }^{44}$ Radiation therapy should be considered in these individuals, with reported improvement in some cases. ${ }^{4}$

Systemic lymphoma in these patients may involve the CNS through invasion of the brain, meninges or spinal cord, causing cerebral syndromes, cranial neuropathies and/or carcinomatous meningitis, and spinal cord compression respectively. In carcinomatous meningitis the CSF may be normal or show mild non-specific abnormalities and the diagnosis can sometimes be made by the use of CSF cytology. ${ }^{43}$ Both radiation therapy and cytotoxic therapy should be considered in these individuals. Although Kaposi's sarcoma is an extremely common malignancy in AIDS, intracerebral Kaposi's sarcoma is exceptionally rare with only two pathologically proven cases having been reported to date. ${ }^{4}$

\section{Vascular involvement (Table I)}

Cerebrovascular syndromes have been described in patients with AIDS and these appear to be primarily the consequence of other pathologies found in these cases. For example, cerebral infarcts are known to occur, secondary to non-bacterial thrombotic endocarditis. ${ }^{3}$ Intracerebral haemorrhage has also been described, resulting from haematological abnormalities such as thrombocytopenia or in some cases from CNS lymphoma. ${ }^{3}$ The possibility of meningovascular syphilis as a cause of cerebrovascular pathology should always be considered in these patients.

\section{PNS complications (Table II)}

A variety of PNS syndromes has been described in AIDS patients. A prominent feature is the development of one of several types of peripheral neuropathy. The most common form is a progressive distal symmetrical sensori-motor neuropathy usually occurring late in the disease in patients with established AIDS.3,7,45 Painful dysaethesias are common but weakness is not prominent and both demyelinating and axonal degenerative processes may be apparent. ${ }^{3,45}$ In the study of 12 homosexual patients with ARC, four of
Table II Neurological complications of HIV infectionPNS complications

Distal sensorimotor neuropathy

Acute, subacute or chronic demyelinating inflammatory neuropathies

Mononeuritis multiplex

Herpes zoster radiculitis and cranial neuropathy

Myopathy and polymyositis

whom subsequently developed AIDS, both mononeuritis multiplex and cranial nerve involvement also occurred in some cases. ${ }^{45}$ There is currently no specific therapy for these neuropathies.

In our own studies of HIV positive patients, several cases with HIV infection alone or ARC have developed inflammatory demyelinating neuropathies which have either been acute or subacute with a Guillain-Barré-like syndrome or a chronic inflammatory demyelinating neuropathy. ${ }^{11}$ Some of these patients have subsequently developed full-blown AIDS. These patients responded favourably to plasmapheresis and/or steroid therapy and the prognosis for this type of neuropathy appears to be good, a number of patients having made a marked recovery. The aetiology is thought to be immune-mediated rather than due to direct HIV infection of the nerves. The occurrence of this neurological complication in patients who do not have full-blown AIDS is reminiscent of the presentation of the AIDS-dementia complex and has important implications for patient diagnosis, screening, handling of body fluids and pathogenesis. ${ }^{11}$

As might be expected from the immunosuppression occurring in AIDS patients herpes zoster radiculitis also occurs with increased frequency in these individuals. The pattern of infection is similar to that occurring in immunocompetent individuals with thoracic segment and trigeminal nerve involvement being prominent. Cranial neuropathy in AIDS may thus result from VZV infection although it may also occur in association with CMV infection. ${ }^{43}$ An additional complication of VZV infection in AIDS patients is dissemination of VZV to the bloodstream and CNS as a consequence of the immunosuppressed state. Treatment of VZV infections is with intravenous acyclovir.

Finally it should be mentioned that myopathy appears to be a complication of AIDS infections. For example, in one series ${ }^{3}$ clinical and pathological features consistent with polymyositis with a necrotizing myopathy occurred in one patient. Both proximal muscle weakness and focal myocarditis have also been described. ${ }^{43,46}$ 


\section{References}

1. Barre-Sinoussi, F., Chermann, J.C., Rey, F. et al., Isolation of a T-lymphotropic retrovirus from a patient at risk for acquired immune deficiency syndrome (AIDS). Science 1983, 220: 868-870.

2. Gallo, R.C., Salahaddin, S.Z., Popovic, M. et al. Frequent detection and isolation of cytopathic retroviruses (HTLV-III) from patients with AIDS and at risk for AIDS. Science 1984, 224: 500-502.

3. Snider, W.D., Simpson, D.M., Nielson, S., Gold, J.W.M., Metroka, C.E. \& Posner, J.B. Neurological complications of acquired immune deficiency syndrome: Analysis of 50 patients. Ann Neurol 1983, 14: 403-418.

4. Levy, R.M., Bredesen, D.E. \& Rosenblum, M.L. Neurological manifestations of the acquired immunodeficiency syndrome (AIDS). Experience at UCSF and review of the literature. $J$ Neurosurg 1985, 62: 475495.

5. Petito, C.K., Cho, E.-S., Lemann, W., Navia, B.A. \& Price, R.W. Neuropathology of acquired immunodeficiency syndrome (AIDS): An autopsy review. $J$ Neuropath Exp Neurol 1986, 45: 635-646.

6. Anders, K., Steinsapir, K.D., Iverson, D.J. et al. Neuropathological findings in the acquired immunodeficiency syndrome (AIDS). Clin Neuropathol 1986, 5: 1-20.

7. Price, R.W. \& Navia, B.A. Infections in AIDS and other immunosuppressed patients. In: Kennedy, P.G.E. \& Johnson, R.T. (eds) Infections of the Nervous System. Butterworths, London, 1987.

8. Lane, H.C., Masur, H., Edgar, L.C., Whalen, G., Rook, A.M. \& Fauci, A.S. Abnormalities of B-cell activation and immunoregulation in patients with the acquired immunodeficiency syndrome. $N$ Engl $\mathrm{J} \mathrm{Med}$ 1983, 309: 453-458.

9. Tucker, T., Dix, R.D., Katzen, C., Davis, R.L. \& Schmidley, J.W. Cytomegalovirus and herpes simplex virus ascending myelitis in a patient with acquired immune deficiency syndrome. Ann Neurol 1985, 18; 74-79.

10. Navia, B.A., Jordan, B.D., Price, R.W. The AIDS dementia complex: I. Clinical features. Ann Neurol 1986, 19: 517-524.

11. Cornblath, D.R., McArthur, J.C., Kennedy, P.G.E., Witte, A.S. \& Griffin, J.W. Inflammatory demyelinating peripheral neuropathies associated with HTLV-III infection. Ann Neurol 1987, 21: 32-40.

12. Belman, A.L., Ultmann, M.H., Horoupian, D. et al. Neurological complications in infants and children with acquired immune deficiency syndrome. Ann Neurol 1985, 18: 560-566.

13. Epstein, L.G., Sharer, L.R., Joshi, V.V., Fojas, M.M., Koenigsberger, M.R. \& Oleske, J.M. Progressive encephalopathy in children with acquired immune deficiency syndrome. Ann Neurol 1985, 17: 488-496.

14. Ho, D.D., Rota, T.R., Schooley, R.T. et al. Isolation of HTLV-III from cerebrospinal fluid and nerve tissues of patients with neurologic syndromes related to the acquired immunodeficiency syndrome. $N$ Engl $\mathrm{J}$ Med 1985, 313: 1493-1497.
15. Resnick, L., Diuarzo-Veronese, F., Schupbach, J. et al Intra-blood-brain-barrier synthesis of HTLV-III specific IgG in patients with neurologic symptoms associated with AIDS or AIDS-related complex. $N$ Engl J Med 1985, 313: 1498-1504.

16. Nielsen, S.L., Petito, C.K., Urmacher, C.D. \& Posner, J.B. Subacute encephalitis in acquired immune deficiency syndrome: A post-mortem study. Am J Clin Pathol 1984, 82: 678-682.

17. Shaw, G.M., Harper, M.E., Hahn, B.H. et al. HTLVIII infection in brains of children and adults with AIDS encephalopathy. Science 1985, 227: 177-182.

18. Levy, J.A., Shimabukuro, J., Hollander, M., Mills, J. \& Kaminsky, L. Isolation of AIDS-associated retroviruses from cerebrospinal fluid and brain of patients with neurological symptoms. Lancet 1985, ii: 586-588.

19. Wiley, C.A., Schrier, R.D., Nelson, J.A., Lampert, P.W. \& Oldstone, M.B.A. Cellular localization of human immunodeficiency virus infection within the brains of acquired immune deficiency syndrome patients. Proc Natl Acad Sci USA 1986, 83: 7089-7093.

20. Pumarola-Sune, T., Navia, B.A., Cordon-Cardo, C., Cho, E.-S., Price, R.W. HIV antigen in the brains of patients with the AIDS dementia complex. Ann Neurol 1987, 21: 490-496.

21. Kennedy, P.G.E., Newsome, D.A., Hess, J. et al. Cytomegalovirus but not human $\mathrm{T}$ lymphotropic virus type III/lymphadenopathy associated virus detected by in situ hybridization in retinal lesions in patients with the acquired immune deficiency syndrome. $\mathrm{Br} \mathrm{Med} J$ 1986, 293: 162-164.

22. Anand, R., Siegal, F., Reed, C., Cheung, T., Forlenza, S. \& Moore, J. Non-cytocidal natural variants of human immunodeficiency virus isolated from AIDS patients with neurological disorders. Lancet 1987, ii: 234-238.

23. Fischl, M. Richman, D.D., Grieco, M.H. et al. The efficacy of azidothymidine (AZT) in the treatment of patients with AIDS and AIDS-related complex. $N$ Engl J Med 1987, 317: 185-191.

24. Kennedy, P.G.E. Neurological complications of varicella-zoster virus infection. In: Kennedy, P.G.E., Johnson, R.T. (eds) Infections of the Nervous System. Butterworths, London, 1987.

25. Pitlik, S.D., Fainstein, V., Bolivar, R. et al. Spectrum of central nervous system complications in homosexual men with acquired immune deficiency syndrome. J Infect Dis 1983, 148: 771-772. (Letter).

26. Dix, R.D., Waitzman, D.M., Follansbee, S. et al. Herpes simplex virus type 2 encephalitis in two homosexual men with persistent lymphadenopathy. Ann Neurol 1985, 17: 203-206.

27. Petito, C., Navia, B.A., Cho, E.-S., Jordan, B.D., George, D.C. \& Price, R.W. Vascular myelopathy pathologically resembling subacute combined degeneration in patients with the acquired immunodeficiency syndrome. $N$ Engl J Med 1985, 312: 874879.

28. Navia, B.A., Cho, E.-S., Petito, C.K. \& Price, R.W. 
The AIDS dementia complex: II. Neuropathology. Ann Neurol 1986, 19: 525-535.

29. Goldstick, L., Mandybur, T.I. \& Bode, R. Spinal cord degeneration in AIDS. Neurology 1985, 35: 103-106.

30. Astrom, K.-E., Mancall, E.L. \& Richardson, E.P. Jr. Progressive multifocal leukoencephalopathy - a hitherto unrecognised complication of chronic lymphatic leukaemia and Hodgkin's disease. Brain 1958, 81: 93-111.

31. Padgett, B.L., Walker, D.L., Zu Rhein, R.M., Hodach, A.E. \& Chou, S.M. JC papovavirus in progressive multifocal leukoencephalopathy. J Infect Dis 1976, 133: 686-690.

32. Weiner, L.P., Herndon, R.M., Narayan, O. et al. Isolation of virus related to SV40 from patients with progressive multifocal leukoencephalopathy. $N$ Engl J Med 1972, 186; 385-390.

33. $\mathrm{Zu}$ Rhein, G.M. Association of papova-virions with a human demyelinating disease (Progressive multifocal leukoencephalopathy). Prog Med Virol 1969, 11: 185247.

34. Rand, K.H., Johnson, K.P., Rubinstein, L.J. et al. Adenine arabinoside in the treatment of progressive multifocal leukoencephalopathy: use of viruscontaining cells in the urine to assess response to therapy. Ann Neurol 1977, 1: 458-462.

35. Pitchenik, A.E., Fischl, M.A. \& Walls, K.W. Evaluation of cerebral-mass lesions in acquired immunodeficiency syndrome. N Engl J Med 1983, 308: 1099 (Letter).

36. Post, M.J.D., Chan, J.C., Hensley, G.T., Hoffman, T.A., Moskowitz, L.B. \& Lippman, S. Toxoplasma encephalitis in Haitian adults with acquired immunodeficiency syndrome: A clinical-pathologicalCT correlation. AJNR 1983, 4: 153-162.

37. Handler, M., Ho, V., Whelan, M. \& Budzilovich, G. Intracerebral toxoplasmosis in patients with acquired immune deficiency syndrome. $J$ Neurosurg 1983, 59: 994-1001.

38. Wiles, C.M. \& Mackenzie, D.W.R. Fungal diseases of the central nervous system. In: Kennedy, P.G.E. \& Johnson, R.T. (eds) Infections of the Nervous System. Butterworths, London, 1987.

39. Snow, R.M. \& Dismukes, W.E. Cryptococcal meningitis. Diagnostic value of cryptococcal antigen in cerebrospinal fluid. Arch Int Med 1975, 135: 11551157.

40. Tjia, T.L., Yeow, Y.K. \& Tan, C.B. Cryptococcal meningitis. J Neurol Neurosurg Psychiatry 1985, 48: 853-858.

41. Diamond, R.D. \& Bennett, J.E. A subcutaneous reservoir for intrathecal therapy of fungal meningitis. N Engl J Med 1973, 288: 186-188.

42. Levy, R.M., Pons, V.G. \& Rosenblum, M.L. Central nervous system mass lesions in the acquired immunodeficiency syndrome (AIDS). J Neurosurg 1984, 61: 9-16.

43. Britton, C.B. \& Miller, J.R. Neurological complications in acquired immunodeficiency syndrome (AIDS). Neurol Clin 1984, 2: 315-339.

44. Fernandez, R., Mouradian, J., Moore, A. \& Asch, A. Malignant lymphoma with central nervous system involvement in six homosexual men with acquired immunodeficiency syndrome. Lab Invest 1983, 48: 25A (Abstract).

45. Lipkin, W.I., Parry, G., Kiprov, D. \& Abrams, D. Inflammatory neuropathy in homosexual men with lymphadenopathy. Neurology 1985, 31: 1479-1483.

46. Britton, C.B., Marquardt, M.D., Koppel, B., Garvey, G. \& Miller, J.R. Neurological complications of the gay immunosuppressed syndrome: clinical and pathological features. Ann Neurol 1982, 12: 80 (Abstract). 